OPEN ACCESS

Edited by:

Luke Henderson,

University of Sydney, Australia

Reviewed by:

Birendra N. Mallick,

Jawaharlal Nehru University, India

Randi Jenssen Hagerman,

UC Davis, United States

*Correspondence:

Ping Zhao

zhaop@sj-hospital.org

Specialty section:

This article was submitted to

Autonomic Neuroscience,

a section of the journal

Frontiers in Physiology

Received: 11 August 2017

Accepted: 11 July 2018

Published: 31 July 2018

Citation:

Wu $Z$ and Zhao $P$ (2018) Epigenetic Alterations in Anesthesia-Induced

Neurotoxicity in the Developing Brain.

Front. Physiol. 9:1024.

doi: 10.3389/fphys.2018.01024

\section{Epigenetic Alterations in Anesthesia-Induced Neurotoxicity in the Developing Brain}

\author{
Ziyi Wu and Ping Zhao* \\ Department of Anesthesiology, Shengjing Hospital of China Medical University, Shenyang, China
}

Before birth and early in life, the developing brain is particularly sensitive to environmental and pharmacological influences. Increasing experimental evidence suggests that an association exists between exposure to anesthesia during a vulnerable period of brain development and subsequent poor neurodevelopmental outcomes. However, the mechanisms underlying this association are not fully understood. Epigenetics, broadly defined as the regulation of gene expression without alterations of DNA sequence, has become a field of tremendous interest in neuroscience. In recent years, a growing body of literature suggests that anesthesia-induced long-term changes in gene transcription and functional deficits in learning and behavior later in life are mediated via epigenetic modifications. This brief review provides an overview of epigenetic mechanisms and highlights the emerging roles played by epigenetic dysfunctions in the processes of anesthesia-induced neurotoxicity in the developing brain. Epigenetic targeting of DNA methyltransferases and/or histone deacetylases may have some therapeutic value. Epigenetics may lead to the identification of novel markers that contribute toward considerable translational significance in the field of neuroprotection.

Keywords: anesthetic agents, developmental neurotoxicity, DNA methylation, histone modification, non-coding RNAs

\section{INTRODUCTION}

Every year, millions of pregnant women, neonates, infants, and toddlers across the world are exposed to anesthesia for surgeries, therapeutic procedures, and imaging studies. However, before birth and early in life, the developing brain is a particularly sensitive target to environmental and pharmacological influences. Currently the majority of general anesthetics used function through $N$-methyl-D-aspartate (NMDA) receptor and/or $\gamma$-aminobutyric acid receptor type A $\left(\mathrm{GABA}_{\mathrm{A}}\right)$ receptor modulation (Garcia et al., 2010; Petrenko et al., 2014). Inhaled and intravenous anesthetics share overlapping effects on these two receptors (Hudson and Hemmings, 2011). Prolonged or excessive stimulation of NMDA receptors and/or $\mathrm{GABA}_{\mathrm{A}}$ receptors may interfere with the neural circuitry during early neurodevelopment, a consequence that may account for the developmental neurotoxicity induced by general anesthetics (Fredriksson et al., 2004; Fredriksson et al., 2007). Accumulating evidence from rodent and primate studies has demonstrated that in utero [most general anesthetics can cross the placenta and reach fetal blood (Palanisamy, 2012)] or neonatal exposure to commonly used inhaled and intravenous general anesthetics is associated with neural degeneration and subsequent neurocognitive impairments, manifested in learning and memory disabilities (Andropoulos and Greene, 2017). Several retrospective clinical 
studies have demonstrated that childhood exposure to general anesthetics pose an increased risk of neurocognitive impairments (Creeley, 2016). Anesthesia-induced toxic effects on the central nervous system attract wide public attention but their underlying mechanisms are largely unknown. An increasing number of studies demonstrate that general anesthetics may initiate abnormal neurodevelopment, at least in part, through epigenetic mechanisms.

Epigenetics refers to the study of heritable changes in the expression and function of genes without alterations in DNA sequence. Major epigenetic mechanisms include DNA methylation, histone modification, and non-coding RNAs. Epigenetics, which acts as a mediator between genotype and environment, plays significant roles in brain development and cognitive processes by translating environmental cues into changes in the expression of target genes (Van Soom et al., 2014; Kundakovic and Champagne, 2015). Emerging studies have revealed that epigenetic dysregulation is one of the hallmarks of abnormal brain function and neurodegenerative diseases (Delgado-Morales et al., 2017; Tran and Miyake, 2017). It is especially interesting that epigenetic dysregulation currently garners much attention as a pivotal player in anesthesia-induced neurotoxicity at the early stages of brain development.

\section{EPIGENETIC CHANGES AND THERAPEUTIC APPROACHES FOR ANESTHETIC-INDUCED DEVELOPMENTAL NEUROTOXICITY}

\section{DNA Methylation and DNMTs Inhibitors}

DNA methylation (Moore et al., 2013; Heyward and Sweatt, 2015) is the most characterized epigenetic event in which DNA methyltransferases (DNMTs) catalyze the covalent conversion of cytosine residues to 5-methylcytosine residues, which can lead to long-term down-regulation of target genes. Three active mammalian DNMTs have been identified, DNMT1, DNMT3a, and DNMT3b. DNMT1 has a preference for hemimethylated DNA, whereas DNMT3a and DNMT3b are involved in the formation of new methylation patterns to unmodified DNA, called de novo DNA methylation. DNA methylation regulates gene expression by recruiting proteins involved in gene repression and/or by blocking promoter regions to which activating transcription factors should bind. DNMT inhibitors are widely used as epigenetic modulators, thereby representing promising targets in epigenetic therapies. DNMT inhibitors can modulate aberrant DNA methylation pattern in a reversible manner by inhibiting DNMT activity.

A recent study has demonstrated that the expression of DNMT1 is significantly increased in the hippocampi of rats with neonatal exposure to isoflurane (Wu et al., 2016). A further chromatin immunoprecipitation (ChIP) study has revealed increased occupancy and methylation (5'-cytosine) levels at the promoter region of the neurotrophin, brainderived neurotrophic factor (BDNF) gene, a critical modulator of synaptic plasticity. This increased methylation at the BDNF promoter region was associated with suppression of BDNF expression and subsequent memory loss. Hippocampal DNMT3a and DNMT3b levels are increased in a rat model with repeated neonatal sevoflurane exposure, resulting in the hypermethylation of BDNF and Reelin genes (Ju et al., 2016). Interestingly, DNMT1 levels do not significantly change (Ju et al., 2016). Different model species, anesthetics, and/or exposure doses within these studies may account for these findings. Pretreatment with the DNMT inhibitor, 5-aza-2'-deoxycytidine, reverses sevofluraneinduced dendritic spine decreases and cognitive abnormalities by inhibiting DNMT activity and enhancing the expression of synaptic plasticity-related genes (Ju et al., 2016).

\section{Histone Modifications and HDAC Inhibitors}

There have been some important publications in recent years that have pointed out the importance of histone modifications in neural development and brain function (Keverne, 2014; Sen, 2015). Histone modifications encompass a vast variety of posttranslational modifications to the tails of histone proteins, and these give rise to varying cellular outcomes. In particular, histone modification by acetylation, which involves the addition of an acetyl group to lysine residues present at the $\mathrm{N}$-terminal tails of the nucleosome, is the most extensively studied one in neuroscience. Generally, acetylated histones are associated with increased transcriptional activity, whereas deacetylated histones are associated with decreased transcriptional activity. Histone acetylation is mediated through histone acetyltransferases (HATs) and histone deacetylases (HDACs), each family comprised of several isoforms. HDACs reverse the activity of HATs and cause a decrease in transcription through the removal of acetyl groups from histone tails. HDACs are typically grouped into four classes: class I HDACs (1-3 and 8), class II HDACs (4-7, 9, and 10), class III HDACs (also known as sirtuins, which are structurally $\mathrm{NAD}^{+}$dependent for enzymatic activity) and class IV HDACs (referred to as HDAC11). Unfortunately, dysregulation of the HATs/HDACs balance may lead to pathologies which have been implicated in anesthesia-induced neurological disorders.

As histone acetylation is typically associated with an increase in the expression of numerous neural genes and in turn, plays an important role in synaptic plasticity, learning and memory, it is generally considered favorable for memory and cognition. As well as enhancing HAT activity, HDAC inhibitors, which are predominantly used as anticancer drugs, have recently been suggested to act as neuroprotective agents and are emerging as powerful cognitive enhancers. HDAC inhibitors are therefore a novel therapy to treat cognitive impairments that are linked to a wide range of neurodegenerative and psychiatric disorders (Graff and Tsai, 2013; Ganai et al., 2016).

In previous studies, rodents exposed to anesthetics during the gestational or neonatal period exhibited long-term developmental neurocognitive abnormalities and alterations in histone acetylation. For example, hippocampal levels of HDAC3 and HDAC8, but not HDAC1 and HDAC2, were elevated in adult rats that were exposed to sevoflurane in the neonatal period. Moreover, sevoflurane-exposed rats 
showed reduced hippocampal levels of acetylated $\mathrm{H} 3 \mathrm{~K} 9 / 14$ and $\mathrm{H} 4 \mathrm{~K} 5 / 12$ and reduced expression of several genes involved in neurodevelopment and neuroplasticity including BDNF, c-Fos, and postsynaptic density protein 95 (Psd-95). It is worth noting that sevoflurane exposure was associated with changes in specific brain regions, such as decreased $\mathrm{H} 3 \mathrm{~K} 9$ and $\mathrm{H} 4 \mathrm{~K} 5 / 12$ acetylation in the hippocampal CA1 region, as well as decreased $\mathrm{H} 2 \mathrm{~K} 14$ acetylation in both that hippocampal CA1 and dentate gyrus (DG) regions. Moreover, impaired hippocampus-dependent spatial and associated memory is observed, rather than explorative behaviors. Upregulation of histone acetylation pharmacologically, using the HDAC inhibitor sodium butyrate $(\mathrm{NaB})$, ameliorated the developmental side effects caused by sevoflurane exposure (Jia et al., 2016). Increased HDAC2 activity and decreased acetylation of $\mathrm{H} 3$ but not $\mathrm{H} 4$ is observed in the hippocampus of isoflurane-exposed rats and in isoflurane-exposed hippocampal neurons, along with decreased histone acetylation of hippocampal neurons in the promoter regions of GLT-1 and mGLuR1/5. NaB improves cognitive impairments in vivo by restoring a decrease in histone acetylation of glutamatergic systems, which has been confirmed in hippocampal neurons (Liang and Fang, 2016). Trichostatin A (TSA) has also been shown to offer protection against neurocognitive impairment and abnormal hippocampal histone acetylation in isoflurane-exposed mice during the neonatal period by enhancing histone acetylation and downstream c-Fos gene expression (Zhong et al., 2015).

The cyclic-AMP-response element binding protein (CREB) signaling pathways have been implicated in anesthesia-induced neurodegenerative changes in basic experimental studies ( $\mathrm{Bi}$ et al., 2016; Ding et al., 2017). CREB-binding protein, also known as $\mathrm{CBP}$, functions by activating transcription, as a co-activator of the transcription factor CREB. CBP is also characterized as a HAT, regulating the degree of histone acetylation via its intrinsic HAT domain. A general anesthetic (a sedative dose of midazolam followed by a combination of nitrous oxide and isoflurane) causes fragmentation of CBP with decrease in its HAT activity. Hypoacetylated $\mathrm{H} 3$ results in down-regulated transcription and expression of BDNF and c-Fos (Dalla Massara et al., 2016). ChIP assays have revealed that the levels of acetylated $\mathrm{H} 3$ in CREB binding sites at the promoter regions of BDNF and c-Fos genes are decreased in the hippocampus, which in turn inhibits their transcription. Reversal of histone hypoacetylation with $\mathrm{NaB}$ blocks the morphological and functional impairments of neuronal development and synaptic communication observed (Dalla Massara et al., 2016). A decrease in the interaction between CBP and CREB has also been reported in the brains of postnatal mice with isoflurane-induced cognitive impairments,

\section{Anesthesia Exposure \\ DNMTs inhibitors: 5-aza-2-deoxycytidine

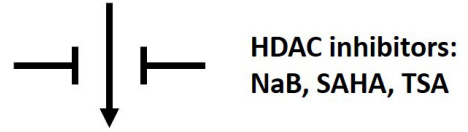 \\ Epigenetic Alterations}

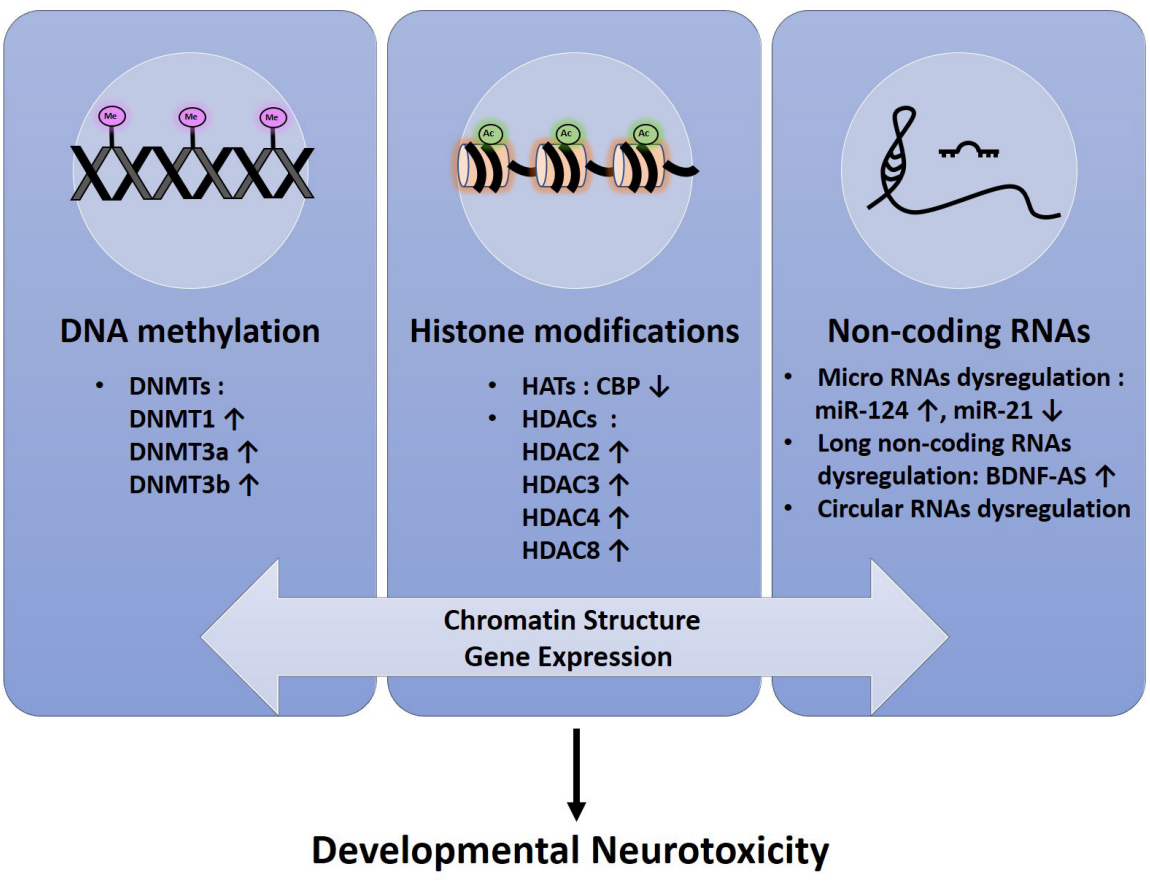

FIGURE 1 | Schematic representation of epigenetic alterations in anesthesia-induced neurotoxicity in the developing brain. 
resulting from an increase in nuclear translocation of HDAC4. HDAC4 interacts with CREB in the nucleus, which results in an impairment in transcriptional activation of CREB and a decrease in the expression levels of BDNF and c-Fos (Sen and Sen, 2016). In an isoflurane-exposed maternal-fetal rat model, overexpression of HDAC2 induced the subsequent downregulation of CREB and was associated with spatial learning and memory impairments in the offspring (Luo et al., 2016). These changes were reversed by suberoylanilide hydroxamic acid (SAHA), a HDAC inhibitor marketed as Vorinostat which is FDA-approved for the treatment of leukemia (Witt et al., 2012), which was administered to the offspring before assessing learning and memory tested by the Morris water maze (Luo et al., 2016).

\section{Non-coding RNAs}

Non-coding RNAs (ncRNAs) (Hombach and Kretz, 2016) represent a large and heterogeneous family of RNA molecules that do not encode proteins. Non-coding RNAs are loosely classified into two major classes: short $(<200$ nucleotides $)$ and long ( $>200$ nucleotides) ncRNAs. The description of multiple kinds of ncRNAs is exponentially increasing and it is now widely accepted that ncRNAs play major biological roles in a myriad of processes, ranging from embryonic development to aging. Micro RNAs (miRNAs) and long non-coding RNAs (lncRNAs) represent the best-characterized of the ncRNAs. Since they function as crucial regulators in gene expression, it is not surprising that dysregulations in miRNAs and/or lncRNAs activity are associated with many complicated human disorders including functional cognitive disorders caused by anesthetics.

Alterations in miRNA and lncRNA activity have been reported after inhaled and/or intravenous anesthetic exposure (Sun and Pei, 2015; Chen et al., 2016; Ye et al., 2016). These alterations may change the expression of related pathological intermediates in anesthetic-induced developmental neurotoxicity, suggesting that ncRNA-based signaling may be a novel target for preventing this neurotoxicity. One example is the neuronal microRNA, miR-124, which is upregulated in ketamine-induced neurodegeneration in mouse hippocampus (Xu et al., 2015). Knocking down miR-124 in vitro reduces ketamine-induced apoptosis in hippocampal CA1 neurons through upregulating $\alpha$-amino-3hydroxy-5-methyl-4-isoxazolepropionic acid (AMPA) receptor phosphorylation and activating PKC/ERK pathway (Xu et al., 2015). Mice subjected to hippocampal miR-124 inhibition in vivo showed improved memory performance (Xu et al., 2015). Another example is miR-21 which is down-regulated in propofol-treated human embryonic stem cell-derived neurons and regulates Sprouty 2 expression (Twaroski et al., 2014). A signal transducer and activator of transcription $3 / \mathrm{miR}$ $21 /$ Sprouty $2 /$ Akt-dependent mechanism is considered to be involved in propofol-induced cell death (Twaroski et al., 2014). BDNF antisense RNA (BDNF-AS) is one discovered functional lncRNA which inhibits the expression of BDNF (Modarresi et al., 2012). BDNF-AS is upregulated in ketamine-injured mouse embryonic neural stem cell-derived neurons, while BDNF is downregulated (Zheng et al., 2016). Downregulation of BDNFAS protects neurons against apoptosis and promotes neurite outgrowth, possibly via the activation of the BDNF-TrkB signaling pathway (Zheng et al., 2016). Additional changes to ncRNAs are also reported in various models of anesthesiainduced neurotoxicity (Jiang et al., 2014; Cao et al., 2015; Sun and Pei, 2016; Song et al., 2017; Zhou et al., 2017).

Additionally, circular RNAs (circRNAs) belong to a new class of ncRNA molecules that are highly abundant in the brain and influence the regulation of gene expression (Hansen et al., 2013). Many circRNAs change their abundance abruptly corresponding to the timing of synaptogenesis (You et al., 2015). Studies have suggested that circRNAs may regulate synaptic plasticity and neuronal function (Szabo et al., 2015; van Rossum et al., 2016). Some studies have provided an insight into the function of circRNAs in neurodegenerative diseases, such as Alzheimer's disease and Parkinson's disease (Floris et al., 2016), and the neurotoxic effects observed in animals exposed to anesthetics. Evidence includes histological changes in neurodegenerative changes. These provide new insights into the possible association of circRNA dysregulation with anesthesia-induced neurotoxicity, although to date the literature is limited. For example, circRNAs can function as miRNA sponges (Hansen et al., 2013) and miRNAs involved in anesthesia-induced neurotoxicity may get inhibited by some unknown circRNAs.

\section{Epigenetic Crosstalk}

In addition to the independent regulation by individual epigenetic mechanisms, it is interesting that an epigenetic crosstalk, i.e., interplay between DNA methylation and histone methylation (Du et al., 2015), may be involved in the processes of modulating disease-associated genomic loci and gene products. Collaborative activities of different epigenetic modifications could result in a common outcome, gene transcription or gene silencing. For example, methyl-CpG-binding protein 2 (MeCP2) is believed to function as a transcriptional repressor by binding to methyl-CpG, recruiting chromatin remodeling proteins, and further suppressing the expression of genes. MeCP2 integrates DNA methylation and histone acetylation at the BDNF gene suppression induced by anesthesia in neonatal rats via enhanced interaction with DNMT1 and HDAC2 (Wu et al., 2016).

\section{CONCLUSION}

So far, conflicting data exist about the effect of anesthetic agents on neurodevelopment in humans and no definite conclusion has been given yet. Although general anesthetics have been considered neuroprotective in pre-clinical studies (Nunes et al., 2013; Zaugg et al., 2014), the effect of anesthesiarelated neurotoxicity remains an area of concern. The most recent studies suggest a novel epigenetic-related mechanism by which anesthetic-induced neuronal toxicity in developing human neurons and animal models (Figure 1). Success of therapeutic intervention using epigenetic modifiers such as DNMT inhibitors and HDAC inhibitors implicates that the epigenetic intervention is promising as potential targeted therapies aimed at mitigating neurotoxic effects of anesthetics in developing brain (Figure 1). 
Further research is needed to fully elucidate the epigenetic basis and its role in this field.

\section{AUTHOR CONTRIBUTIONS}

$\mathrm{PZ}$ and $\mathrm{ZW}$ wrote this review article.

\section{REFERENCES}

Andropoulos, D. B., and Greene, M. F. (2017). Anesthesia and developing brains - implications of the fda warning. N. Engl. J. Med. 376, 905-907. doi: 10.1056/ NEJMp 1700196

Bi, J., Zhang, H., Lu, J., and Lei, W. (2016). Nobiletin ameliorates isofluraneinduced cognitive impairment via antioxidant, anti-inflammatory and antiapoptotic effects in aging rats. Mol. Med. Rep. 14, 5408-5414. doi: 10.3892/mmr. 2016.5919

Cao, S. E., Tian, J., Chen, S., Zhang, X., and Zhang, Y. (2015). Role of miR-34c in ketamine-induced neurotoxicity in neonatal mice hippocampus. Cell Biol. Int. 39, 164-168. doi: 10.1002/cbin.10349

Chen, X., Zhou, X., Lu, D., Yang, X., Zhou, Z., Chen, X., et al. (2016). Aberrantly expressed long noncoding RNAs are involved in sevofluraneinduced developing hippocampal neuronal apoptosis: a microarray related study. Metab. Brain Dis. 31, 1031-1040. doi: 10.1007/s11011-016-9838-6

Creeley, C. E. (2016). From drug-induced developmental neuroapoptosis to pediatric anesthetic neurotoxicity-where are we now? Brain Sci. 6:E32. doi: 10.3390/brainsci6030032

Dalla Massara, L., Osuru, H. P., Oklopcic, A., Milanovic, D., Joksimovic, S. M., Caputo, V., et al. (2016). General anesthesia causes epigenetic histone modulation of c-fos and brain-derived neurotrophic factor, target genes important for neuronal development in the immature rat hippocampus. Anesthesiology 124, 1311-1327. doi: 10.1097/aln.0000000000001111

Delgado-Morales, R., Agis-Balboa, R. C., Esteller, M., and Berdasco, M. (2017). Epigenetic mechanisms during ageing and neurogenesis as novel therapeutic avenues in human brain disorders. Clin. Epigenetics 9:67. doi: 10.1186/s13148017-0365-z

Ding, M. L., Ma, H., Man, Y. G., and Lv, Y. H. (2017). Protective effects of green tea polyphenol, epigallocatechin-3-gallate against sevoflurane-induced neuronal apoptosis involves regulation of CREB -BDNF-Trk-B and PI3K/Akt/mTOR signalling pathways in neonatal mice. Can. J. Physiol. Pharmacol. 95, 13961405. doi: 10.1139/cjpp-2016-0333

Du, J., Johnson, L. M., Jacobsen, S. E., and Patel, D. J. (2015). DNA methylation pathways and their crosstalk with histone methylation. Nat. Rev. Mol. Cell Biol. 16, 519-532. doi: 10.1038/nrm4043

Floris, G., Zhang, L., Follesa, P., and Sun, T. (2016). Regulatory role of circular RNAs and neurological disorders. Mol. Neurobiol. 54, 5156-5165. doi: 10.1007/ s12035-016-0055-4

Fredriksson, A., Archer, T., Alm, H., Gordh, T., and Eriksson, P. (2004). Neurofunctional deficits and potentiated apoptosis by neonatal NMDA antagonist administration. Behav. Brain Res. 153, 367-376. doi: 10.1016/j.bbr. 2003.12.026

Fredriksson, A., Ponten, E., Gordh, T., and Eriksson, P. (2007). Neonatal exposure to a combination of N-methyl-D-aspartate and gamma-aminobutyric acid type a receptor anesthetic agents potentiates apoptotic neurodegeneration and persistent behavioral deficits. Anesthesiology 107, 427-436. doi: 10.1097/01.anes. $0000278892.62305 .9 c$

Ganai, S. A., Ramadoss, M., and Mahadevan, V. (2016). Histone Deacetylase (HDAC) inhibitors - emerging roles in neuronal memory, learning, synaptic plasticity and neural regeneration. Curr. Neuropharmacol. 14, 55-71. doi: 10. 2174/1570159X13666151021111609

Garcia, P. S., Kolesky, S. E., and Jenkins, A. (2010). General anesthetic actions on GABA(A) receptors. Curr. Neuropharmacol. 8, 2-9. doi: 10.2174/ 157015910790909502

Graff, J., and Tsai, L. H. (2013). The potential of HDAC inhibitors as cognitive enhancers. Annu. Rev. Pharmacol. Toxicol. 53, 311-330. doi: 10.1146/annurevpharmtox-011112-140216

\section{FUNDING}

This work was supported by the National Nature Science Foundation of China (No. 81671311), Science and Technology Foundation of Liaoning Province (No. 2015020467), and Outstanding Scientific Fund of Shengjing Hospital (No. 201708).

Hansen, T. B., Jensen, T. I., Clausen, B. H., Bramsen, J. B., Finsen, B., Damgaard, C. K., et al. (2013). Natural RNA circles function as efficient microRNA sponges. Nature 495, 384-388. doi: 10.1038/nature11993

Heyward, F. D., and Sweatt, J. D. (2015). DNA methylation in memory formation: emerging insights. Neuroscientist 21, 475-489. doi: 10.1177/1073858415579635

Hombach, S., and Kretz, M. (2016). Non-coding RNAs: classification. Adv. Exp. Med. Biol. 937, 3-17. doi: 10.1007/978-3-319-42059-2_1

Hudson, A. E., and Hemmings, H. C. Jr. (2011). Are anaesthetics toxic to the brain? Br. J. Anaesth. 107, 30-37. doi: 10.1093/bja/aer122

Jia, M., Liu, W. X., Yang, J. J., Xu, N., Xie, Z. M., Ju, L. S., et al. (2016). Role of histone acetylation in long-term neurobehavioral effects of neonatal exposure to sevoflurane in rats. Neurobiol. Dis. 91, 209-220. doi: 10.1016/j.nbd.2016.03.017

Jiang, X. L., Du, B. X., Chen, J., Liu, L., Shao, W. B., and Song, J. (2014) microRNA-34a negatively regulates anesthesia-induced hippocampal apoptosis and memory impairment through FGFR1. Int. J. Clin. Exp. Pathol. 7, 6760-6767.

Ju, L. S., Jia, M., Sun, J., Sun, X. R., Zhang, H., Ji, M. H., et al. (2016). Hypermethylation of hippocampal synaptic plasticity-related genes is involved in neonatal sevoflurane exposure-induced cognitive impairments in rats. Neurotox. Res. 29, 243-255. doi: 10.1007/s12640-015-95851

Keverne, E. B. (2014). Significance of epigenetics for understanding brain development, brain evolution and behaviour. Neuroscience 264, 207-217. doi: 10.1016/j.neuroscience.2012.11.030

Kundakovic, M., and Champagne, F. A. (2015). Early-life experience, epigenetics, and the developing brain. Neuropsychopharmacology 40, 141-153. doi: 10.1038/ npp.2014.140

Liang, B., and Fang, J. (2016). Postnatal isoflurane exposure induces cognitive impairment and abnormal histone acetylation of glutamatergic systems in the hippocampus of adolescent rats. J. Mol. Neurosci. 60, 11-20. doi: 10.1007/ s12031-016-0756-1

Luo, F., Hu, Y., Zhao, W., Zuo, Z., Yu, Q., Liu, Z., et al. (2016). Maternal exposure of rats to isoflurane during late pregnancy impairs spatial learning and memory in the offspring by up-regulating the expression of histone deacetylase 2. PLoS One 11:e0160826. doi: 10.1371/journal.pone.0160826

Modarresi, F., Faghihi, M. A., Lopez-Toledano, M. A., Fatemi, R. P., Magistri, M., Brothers, S. P., et al. (2012). Inhibition of natural antisense transcripts in vivo results in gene-specific transcriptional upregulation. Nat. Biotechnol. 30, 453459. doi: $10.1038 /$ nbt. 2158

Moore, L. D., Le, T., and Fan, G. (2013). DNA methylation and its basic function. Neuropsychopharmacology 38, 23-38. doi: 10.1038/npp.2012.112

Nunes, R. R., Duval Neto, G. F., de Alencar, J. C., Franco, S. B., de Andrade, N. Q., Dumaresq, D. M., et al. (2013). Anesthetics, cerebral protection and preconditioning. Rev. Braz. Anesthesiol. 63, 119-128. doi: 10.1016/j.bjane.2012. 06.003

Palanisamy, A. (2012). Maternal anesthesia and fetal neurodevelopment. Int. J. Obstet. Anesth. 21, 152-162. doi: 10.1016/j.ijoa.2012.01.005

Petrenko, A. B., Yamakura, T., Sakimura, K., and Baba, H. (2014). Defining the role of NMDA receptors in anesthesia: are we there yet? Eur. J. Pharmacol. 723, 29-37. doi: 10.1016/j.ejphar.2013.11.039

Sen, N. (2015). Epigenetic regulation of memory by acetylation and methylation of chromatin: implications in neurological disorders, aging, and addiction. Neuromolecular Med. 17, 97-110. doi: 10.1007/s12017-0148306-x

Sen, T., and Sen, N. (2016). Isoflurane-induced inactivation of CREB through histone deacetylase 4 is responsible for cognitive impairment in developing brain. Neurobiol. Dis. 96, 12-21. doi: 10.1016/j.nbd.2016.08.005

Song, C., Song, C., Chen, K., and Zhang, X. (2017). Inhibition of long non-coding RNA IGF2AS protects apoptosis and neuronal loss in anesthetic-damaged 
mouse neural stem cell derived neurons. Biomed. Pharmacother. 85, 218-224. doi: 10.1016/j.biopha.2016.10.094

Sun, W., and Pei, L. (2015). microRNA expression profiling of propofol-treated developing rat hippocampal astrocytes. DNA Cell Biol. 34, 511-523. doi: 10. 1089/dna.2015.2831

Sun, W. C., and Pei, L. (2016). rno-miR-665 targets BCL2L1 (Bcl-xl) and increases vulnerability to propofol in developing astrocytes. J. Neurochem. 138, 233-242. doi: $10.1111 /$ jnc. 13647

Szabo, L., Morey, R., Palpant, N. J., Wang, P. L., Afari, N., Jiang, C., et al. (2015). Statistically based splicing detection reveals neural enrichment and tissuespecific induction of circular RNA during human fetal development. Genome Biol. 16:126. doi: 10.1186/s13059-015-0690-5

Tran, N. Q. V., and Miyake, K. (2017). Neurodevelopmental disorders and environmental toxicants: epigenetics as an underlying mechanism. Int. J. Genomics 2017:7526592. doi: 10.1155/2017/7526592

Twaroski, D. M., Yan, Y., Olson, J. M., Bosnjak, Z. J., and Bai, X. (2014). Downregulation of microRNA-21 is involved in the propofol-induced neurotoxicity observed in human stem cell-derived neurons. Anesthesiology 121, 786-800. doi: 10.1097/aln.0000000000000345

van Rossum, D., Verheijen, B. M., and Pasterkamp, R. J. (2016). Circular RNAs: novel regulators of neuronal development. Front. Mol. Neurosci. 9:74. doi: 10.3389/fnmol.2016.00074

Van Soom, A., Peelman, L., Holt, W. V., and Fazeli, A. (2014). An introduction to epigenetics as the link between genotype and environment: a personal view. Reprod. Domest. Anim. 49(Suppl. 3), 2-10. doi: 10.1111/rda.12341

Witt, O., Milde, T., Deubzer, H. E., Oehme, I., Witt, R., Kulozik, A., et al. (2012). Phase I/II intra-patient dose escalation study of vorinostat in children with relapsed solid tumor, lymphoma or leukemia. Klin. Padiatr. 224, 398-403. doi: 10.1055/s-0032-1323692

Wu, J., Bie, B., and Naguib, M. (2016). Epigenetic manipulation of brainderived neurotrophic factor improves memory deficiency induced by neonatal anesthesia in rats. Anesthesiology 124, 624-640. doi: 10.1097/aln. 0000000000000981

Xu, H., Zhang, J., Zhou, W., Feng, Y., Teng, S., and Song, X. (2015). The role of miR-124 in modulating hippocampal neurotoxicity induced by ketamine anesthesia. Int. J. Neurosci. 125, 213-220. doi: 10.3109/00207454.2014.919915
Ye, J., Zhang, Z., Wang, Y., Chen, C., Xu, X., Yu, H., et al. (2016). Altered hippocampal microRNA expression profiles in neonatal rats caused by sevoflurane anesthesia: microRNA profiling and bioinformatics target analysis. Exp. Ther. Med. 12, 1299-1310. doi: 10.3892/etm.2016. 3452

You, X., Vlatkovic, I., Babic, A., Will, T., Epstein, I., Tushev, G., et al. (2015). Neural circular RNAs are derived from synaptic genes and regulated by development and plasticity. Nat. Neurosci. 18, 603-610. doi: 10.1038/nn. 3975

Zaugg, M., Lucchinetti, E., Behmanesh, S., and Clanachan, A. S. (2014). Anesthetic cardioprotection in clinical practice from proof-of-concept to clinical applications. Curr. Pharm. Des. 20, 5706-5726. doi: 10.2174/ 1381612820666140204120829

Zheng, X., Lin, C., Li, Y., Ye, J., Zhou, J., and Guo, P. (2016). Long noncoding RNA BDNF-AS regulates ketamine-induced neurotoxicity in neural stem cell derived neurons. Biomed. Pharmacother. 82, 722-728. doi: 10.1016/j.biopha. 2016.05.050

Zhong, T., Guo, Q., Zou, W., Zhu, X., Song, Z., Sun, B., et al. (2015). Neonatal isoflurane exposure induces neurocognitive impairment and abnormal hippocampal histone acetylation in mice. PLoS One 10:e0125815. doi: 10.1371/ journal.pone.0125815

Zhou, X., Xian, D., Xia, J., Tang, Y., Li, W., Chen, X., et al. (2017). MicroRNA$34 \mathrm{c}$ is regulated by $\mathrm{p} 53$ and is involved in sevoflurane-induced apoptosis in the developing rat brain potentially via the mitochondrial pathway. Mol. Med. Rep. 15, 2204-2212. doi: 10.3892/mmr.2017.6268

Conflict of Interest Statement: The authors declare that the research was conducted in the absence of any commercial or financial relationships that could be construed as a potential conflict of interest.

Copyright (c) $2018 \mathrm{Wu}$ and Zhao. This is an open-access article distributed under the terms of the Creative Commons Attribution License (CC BY). The use, distribution or reproduction in other forums is permitted, provided the original author(s) and the copyright owner(s) are credited and that the original publication in this journal is cited, in accordance with accepted academic practice. No use, distribution or reproduction is permitted which does not comply with these terms. 